\title{
REDUCED PRODUCTS, HORN SENTENCES, AND DECISION PROBLEMS
}

\author{
BY FRED GALVIN ${ }^{1}$ \\ Communicated by D. Scott, September 1, 1966
}

We shall consider a first order language with equality, and the corresponding structures. Reduced products (powers) and Horn sentences are defined in [4, pp. 199, 211]. An $\forall_{n}$ sentence is a prenex sentence whose prefix consists of a block of universal quantifiers, followed by a block of existential quantifiers, followed by another block of universal quantifiers, and so on $n$ times; $\exists_{n}$ sentences are defined dually. An $E C\left(H C, \forall_{n} C\right)$ is the class of all models of an elementary sentence (Horn sentence, $\forall_{n}$ sentence), and an $E C_{\Delta}\left(H C_{\Delta}, \forall_{n} C_{\Delta}\right)$ is the class of all models of a set of elementary sentences (Horn sentences, $\forall_{n}$ sentences).

THEOREM 1. A sentence is preserved by proper reduced products iff it is equivalent to a Horn sentence.

The "if" part of Theorem 1 is due to Chang [4, Theorem 2.6, p. 215]. The "only if" was conjectured by Chang [8, p. 307] and proved by Keisler [8, result A, p. 307; Corollary 4.3, p. 322] assuming that at least one case of the generalized continuum hypothesis holds, i.e., that $2^{\alpha}=\alpha^{+}$for some infinite cardinal $\alpha$. Now, I have not found a direct proof of Theorem 1 without using the continuum hypothesis. Instead I have observed that, using Ershov's results in [2], one can eliminate the continuum hypothesis from Theorem 1 by Kreisel's method [11, p. 165] based on Gödel's proof of the consistency of the continuum hypothesis. On the basis of Theorem 1, several related results (Theorems 2, 6, and 8), previously known to follow from the continuum hypothesis, can be proved without assuming that hypothesis and without further recourse to Kreisel's method.

THEOREM 2. An EC $C_{\Delta}$ is closed under proper reduced products iff it is an $H C_{\Delta}$.

The "if" in Theorem 2 is due to Chang [4, Corollary 2.7, p. 215]; the "only if" was proved by Keisler [8, Corollary 4.1, p. 322] assum-

\footnotetext{
1 Most of these results are from the author's thesis, supervised by Professor Bjarni Jonsson at the University of Minnesota, with support from NSF grant GP-1612. Other results were obtained and this paper was prepared under NSF grant GP-4608 at the University of California, under the direction of Professors Alfred Tarski and Leon Henkin.
} 
ing that $2^{\alpha}=\alpha^{+}$for some infinite cardinal $\alpha$ not less than the number of sentences needed to characterize the given $E C_{\Delta}$. Since a conjunction of Horn sentences is equivalent to a Horn sentence, it is clear that an $E C$ is an $H C_{\Delta}$ iff it is an $H C$; hence Theorem 1 is included in Theorem 2.

The elementary types of Boolean algebras were first described by Tarski [12]. We will use Ershov's notation [2, p. 22] for these types; thus the type of a nontrivial atomless Boolean algebra is $(0,0,1)$. We will say that a class $K$ of structures is closed under $(n, p, q)$ powers if the reduced power $A^{I} / D$ belongs to $K$ for any $A \in K$ and any filter $D$ such that the Boolean algebra $2^{I} / D$ is of type $(n, p, q)$. We will say that $K$ is closed under direct products if it is closed under direct products of two factors, i.e., if the direct product $A \times B$ belongs to $K$ whenever $A$ and $B$ belong to $K$. Vaught has shown [3, Corollary 6.7 , p. 84] that an $E C_{\Delta}$ closed under direct products of two factors is also closed under direct products of arbitrary nonempty families of structures. The next theorem is a Vaught-type result for reduced products: it tells us that classes of structures defined in certain ways, and closed under certain special types of reduced product, are closed under arbitrary proper reduced products.

Theorem 3. Suppose $K$ is one of the following:

(a) an $\forall_{2} C_{\Delta}$ closed under direct products;

(b) an $\forall_{4} C_{\Delta}$ closed under direct products and $(0,0,1)$-powers;

(c) an $\forall_{5} C_{\Delta}$ closed under direct products, $(0,0,1)$-powers, and $(1,0,1)$-powers;

(d) an $\forall_{6} C_{\Delta}$ closed under direct products, $(0,0,1)$-powers, and $(1,1,0)$-powers;

(e) an $\forall_{9} C_{\Delta}$ closed under direct products, $(0,0,1)$-powers, $(1,1,0)$ powers, and $(1,0,1)$-powers;

(f) an $E C_{\Delta}$ closed under direct products, $(0,0,1)$-powers, $(1,1,0)$ powers, $(1,0,1)$-powers, and $(2,1,0)$-powers.

Then $K$ is closed under proper reduced products, and is therefore an $H C_{\Delta}$.

Theorems 3(a)-(e) all become false, even for classes of Boolean algebras, if $\forall_{n} C_{\Delta}$ is changed to $\exists_{n} C$; for case (a) this was shown by Chang and Morel [1, p. 153]. 3(a) is essentially due to Weinstein, who showed [13, Theorem 1.3.1, p. 23] that an $\forall_{2}$ sentence preserved by direct products is equivalent to an $\forall_{2}$ Horn sentence; moreover, it follows easily by the methods of [13] that a set of $\forall_{2}$ sentences which is preserved by direct products is equivalent to a set of $\forall_{2}$ Horn sentences, and is therefore preserved by proper reduced products. 
Weinstein has remarked that the elementary type of a reduced power $A^{I} / D$ depends only on the elementary types of the structure $A$ and the Boolean algebra $2^{I} / D$; i.e., if $A \equiv B$ and $2^{I} / D \equiv 2^{J} / E$, then $A^{I} / D \equiv B^{J} / E$. (One can derive this from the basic theorem of Feferman and Vaught $[3$, Theorem 3.1, p. 65] on generalized products, using a result of Ershov's [2, Theorem 5, p. 28] on the theory of filters; alternatively, one can give a direct proof, similar to the proof of the Feferman-Vaught theorem.) Now let $K$ be an $E C_{\Delta}$, and consider any filter $D$ over any set $I$ such that the Boolean algebra $2^{I} / D$ is of type $(n, p, q)$. In view of Weinstein's observation, $K$ is closed under $(n, p, q)$-powers iff $A^{I} / D \in K$ for all $A \in K$. Moreover, Ershov has shown $[2$, p. 31] that every Boolean algebra is elementarily equivalent to one of the form $2^{\omega} / D$. Consequently, in order to test whether an $E C_{\Delta}$ is closed under $(n, p, q)$-powers, it suffices to consider a single filter over $\omega$. Thus we obtain from $3(\mathrm{~b})$ and $3(\mathrm{f})$ the following corollaries:

Corollary 4. Let $D_{0}$ be the filter of cofinite subsets of $\omega$. If $K$ is an $\forall_{4} C_{\Delta}$, and if $A \times B \in K$ and $A^{\omega} / D_{0} \in K$ for all $A, B \in K$, then $K$ is an $H C_{\Delta}$.

Corollary 5. There are four filters $D_{0}, D_{1}, D_{2}, D_{3}$ over $\omega$ such that any class $K$ of structures is an $H C_{\Delta}$ iff it satisfies the following conditions:

(a) $K \in E C_{\Delta}$;

(b) if $A, B \in K$, then $A \times B \in K$;

(c) if $A \in K$, then $A^{\omega} / D_{i} \in K$ for $i=0,1,2,3$.

In particular, an $E C_{\Delta}$ that is closed under direct products (of two factors) and $\omega$-indexed proper reduced powers is an $H C_{\Delta}$. This improves on some results of Keisler's: (I) If $2^{\omega}=\omega^{+}$, and if $K$ is the class of all models of a countable set of sentences, and $K$ is closed under $\omega$-indexed proper reduced products, then $K$ is an $H C_{\Delta}$ [8, Corollary 4.1, p. 322]. (II) If $2^{\alpha}=\alpha^{+}$for some infinite cardinal $\alpha$, then any sentence preserved by direct products and proper reduced powers is equivalent to a Horn sentence [6, Theorem 4]. (III) If $2^{\omega}=\omega^{+}$, then any sentence preserved by direct products and $\omega$-indexed proper reduced powers is equivalent to a Horn sentence. (This follows from (II) and [8, Corollary 4.2, p. 322].)

TheOREM 6. An EC $C_{\Delta}$ is closed under proper reduced powers iff it is characterized by a set of disjunctions of Horn sentences.

This was proved by Keisler [8, Corollary 4.2, p. 322] assuming that $2^{\alpha}=\alpha^{+}$for some infinite cardinal $\alpha$ not less than the number of sen- 
tences needed to characterize the given $E C_{\Delta}$. Note that, by the remarks preceding Corollary 4 , an $E C_{\Delta}$ that is closed under $\omega$-indexed proper reduced powers is closed under arbitrary proper reduced powers.

CoRollary 7. A sentence is preserved by proper reduced powers iff it is equivalent to a disjunction of Horn sentences.

This was proved by Keisler [8, Corollary 4.3, p. 322] assuming that $2^{\alpha}=\alpha^{+}$for some infinite cardinal $\alpha$.

THEOREM 8. If every Horn sentence holding in all members of a class $K$ of structures holds in the structure $B$, then some elementary extension of $B$ is isomorphic to a proper reduced product of members of $K$.

A stronger form of Theorem 8, with "ultrapower" in place of "elementary extension," was proved by Keisler [8, result B, p. 308] assuming the generalized continuum hypothesis. Note that this stronger result follows from Theorem 8 together with Keisler's theorem to the effect that (assuming the generalized continuum hypothesis) elementarily equivalent structures have isomorphic ultrapowers [7, Theorem 2.4, p. 487]. Theorem 1 can be strengthened to the following "interpolation theorem," from which Theorems 2 and 6 can also be derived:

THEOREM 9. Given a sentence $\phi$, we can effectively find finitely many Horn sentences $\phi_{1}, \cdots, \phi_{n}$ with the property that, if $K$ is any class of structures closed under proper reduced products, then $K \vDash \phi$ iff $K \vDash \phi_{i}$ for some $i$.

For example, if $\phi=\forall x \exists y[(P x y \wedge \neg Q x y) \vee R x y]$, we can take $\phi_{1}$ $=\forall x \exists y R x y$ and $\phi_{2}=\forall x \exists y_{1} y_{2}\left[P x y_{1} \wedge\left(Q x y_{1} \rightarrow R x y_{2}\right)\right]$. In this case, if $K$ is even closed under direct products, we have that $K \vDash \phi$ iff either $K \vDash \phi_{1}$ or $K \vDash \phi_{2}$. By the way, Theorem 9 remains true if we change "proper reduced products" and "Horn sentences" to "direct products" and "product sentences" (i.e., sentences preserved by direct products); this fact was discovered independently by Weinstein and myself, and is proved in [13]. The next two theorems answer an unpublished question of Addison's, as to whether there are any natural classes of sentences with the property of generating, up to logical equivalence, under Boolean operations, all elementary sentences.

THEOREM 10. Every sentence is equivalent to a Boolean combination of Horn sentences.

For example, if $\phi=\forall x \exists y[(P x y \wedge \neg Q x y \wedge \neg R x y) \vee S x y]$, then 
$\left.\phi \Leftrightarrow \phi_{1} \wedge\right\rceil \phi_{2}$, where

$$
\phi_{1}=\forall x y_{0} \exists y_{1} y_{2}\left[\left(P x y_{0} \rightarrow P x y_{1}\right) \wedge\left(Q x y_{1} \rightarrow S x y_{2}\right) \wedge\left(R x y_{1} \rightarrow S x y_{2}\right)\right]
$$

and $\phi_{2}=\exists x \forall y[\neg P x y \wedge \neg S x y]$. For amusement, the reader may try to express the sentence

$$
\forall x \exists y[P x y \vee Q x y \quad \vee(\neg R x y \wedge \neg S x y)]
$$

as a Boolean combination of Horn sentences. The definition of a strict Horn sentence may be obtained from the definition of a Horn sentence [4, p. 211] by changing "at most one" to "exactly one"; equivalently, a strict Horn sentence is a Horn sentence that holds in the "unit system" [4, p. 198]. It is easy to see that every Horn sentence is equivalent to a Boolean combination of (at most two) strict Horn sentences. Hence Theorem 10 can be slightly improved: Every sentence is equivalent to a Boolean combination of strict Horn sentences.

THEOREM 11. Every sentence is equivalent to a Boolean combination of sentences preserved by direct factors.

A syntactical characterization of sentences preserved by direct factors has been given by Keisler [9, Corollary 6.2, p. 348].

Corollary 12. If $A \equiv A \times B \times C$, then $A \equiv A \times B$.

This corollary is also easily derivable from a result of Vaught's $[3$, Theorem 6.6, p. 83]. Note that the corollary becomes false if elementary equivalence is replaced by isomorphism; Kinoshita has given a counterexample using countable Boolean algebras [10]. A simpler proof of Kinoshita's result may be found in [5].

Theorem 13. $\left(\prod_{i \in I} A_{i} / D\right)^{J} / E \equiv \prod_{i \in I}\left(A_{i}^{J} / E\right) / D$, for any structures $A_{i}$ and any filters $D$ and $E$.

Corollary 14. $\left(A^{I} / D\right)^{J} / E \equiv\left(A^{J} / E\right)^{I} / D$.

On the other hand, the structures $\left(A^{I} / D\right)^{J} / E$ and $\left(A^{J} / E\right)^{I} / D$ need not be isomorphic, nor even have the same cardinality [4, p. 207]. By a Horn theory I mean the set of all consequences of a set of Horn sentences; equivalently, a Horn theory is the theory of a class of structures closed under proper reduced products. (The equivalence follows from Theorem 8 or Theorem 9.)

ThEOREM 15. If $T$ is either (a) a complete theory or (b) a Horn theory, the decision problem for validity in $T$ is reducible to the same problem restricted to Horn sentences. 
This follows from Theorem 10 in the case of a complete theory, and from Theorem 9 in the case of a Horn theory. 15(b) generalizes Ershov's result [2, p. 17] that, if a class $K$ of structures has a decidable theory, so does the class of all reduced products of members of $K .15$ (b) can also be regarded as a generalization of Tarski's result [12] that the theory of Boolean algebras is decidable, since it is easy to see that every Horn sentence holding in the 2-element Boolean algebra holds in all nontrivial Boolean algebras. (Of course this follows from the above-mentioned result of Ershov's, that every Boolean algebra is elementarily equivalent to a reduced power of the 2element Boolean algebra; but there is also a simple direct proof.) However, we do not have a new proof of Tarski's result, since the decidability of the theory of Boolean algebras was needed to get effectiveness in Theorem 9.

\section{REFERENCES}

1. C. C. Chang and Anne C. Morel, On closure under direct product, J. Symbolic Logic 23 (1958), 149-154.

2. Yu. L. Ershov, Decidability of the elementary theory of relatively complemented distributive lattices and the theory of filters, Algebra i Logika Sem. 3 (1964), 17-38. (Russian)

3. S. Feferman and R. L. Vaught, The first order properties of products of algebraic systems, Fund. Math. 47 (1959), 57-103.

4. T. Frayne, A. C. Morel and D. S. Scott, Reduced direct products, Fund. Math. 51 (1962), 195-228.

5. Paul R. Halmos, Lectures on Boolean algebras, Van Nostrand Mathematical Studies No. 1, Van Nostrand, Princeton, N. J., 1963.

6. H. Jerome Keisler, Properties preserved under reduced products. II, Amer. Math. Soc. Notices 8 (1961), 64.

7. - Ultraproducts and elementary classes, Indag. Math. 23 (1961), 477-495.

8. - Reduced products and Horn classes, Trans. Amer. Math. Soc. 117 (1965), $307-328$.

9. - Some applications of infinitely long formulas, J. Symbolic Logic 30 (1965), 339-349.

10. Shin'ichi Kinoshita, $A$ solution of a problem of $R$. Sikorski, Fund. Math. 40 (1953), 39-41.

11. G. Kreisel, Some uses of metamathematics, British J. Philos. Sci. 7 (1956), 161-173.

12. Alfred Tarski, Arithmetical classes and types of Boolean algebras, Preliminary report, Bull. Amer. Math. Soc. 55 (1949), 64.

13. Joseph M. Weinstein, First order properties preserved by direct product, Ph.D. Thesis, University of Wisconsin, Madison, Wis., 1965.

University of California, Berkeley 